\title{
Application of Sensorimotor Rhythm on Sport Performance Enhancement
}

\author{
Ming-Yang Cheng* \\ Cluster of Excellence Cognitive Interaction Technology (CITEC), Bielefeld University, Germany \\ *Corresponding author: Ming-Yang Cheng, Cluster of Excellence Cognitive Interaction Technology (CITEC), Bielefeld University, Germany \\ Submission: 海 September 21, 2017; Published: 海 October 17, 2017
}

\section{Opinion}

Neurofeedback training (NFT) offers insight into how the mind works. NFT is a technique to maintain electroencephalography (EEG) within an optimal zone that has been related to a desirable behavior by the audio or visual feedback in real time. The basic assumption of EEG NFT is: change the EEG, change the behavior [1].

Sensorimotor rhythm (SMR) is a special category of EEG frequency observed in the motor cortex and the activity is inversely related to the activity of the sensorimotor cortex [2]. Higher SMR power is associated with the improved processing efficiency in superior performance. Recent evidence has shown that higher SMR power reflects the superior dart-throwing performance in skilled dart throwing players compared to novices [3]. Similarly, Cheng et al. [4] investigated personal best and worst pistol shooting performances and found that the best performances were preceded by higher SMR power compared to the worst performances.

In the direction of NFT in sport performance, augmented SMR power shows beneficial effects on golf putting performance enhancement. Cheng at al. [5] reported that pre-elite golfers improved the golf putting performance after eight times of SMR NFT, together with an increased SMR activity prior to putting execution, suggesting augmented SMR NFT may result in an improved regulation of somatosensory and sensorimotor pathways, which may lead to more efficient psychomotor [6].

Several theoretical issues should be considered for the future studies. Firstly, investigation on the frequency band specificity. Adjacent frequency bands can be altered by NFT as well [7]. Cheng et al. [5] were able to demonstrate the frequency band specificity of NFT by showing the lack of significant change on flanking frequency bands (theta, alpha, low beta, and high beta) after eight sessions of augmented SMR NFT in a golf putting task. However, the differences in the type of learning, attention, motivation, effort, reinforcement monitoring, etc., which may affect the activity of flanking bands during training. Hence, future studies are recommended to record and report the EEG based on a wider spectrum recording, which can further our understanding of the effects caused by the NFT on adjacent frequency bands. Secondly, the topographical specificity.
The concurrent change of cortical activity in areas other than the training site after NFT is equally important to see whether behavioral changes are closely linked to the changes generated solely from the training site. Cheng et al. [5] found a concurrent change in Pz after eight sessions of augmented SMR training at $\mathrm{Cz}$ for putting performance, suggesting the NFT training targeting to reduce activation of the sensorimotor cortex has spillover to affect the parietal region. It is recommended that future NFT studies examine cortical activity at not only the training site but also at adjacent sites and other more remote regions of the brain so that the cortical mechanism underlying NFT may be further understood.

Other issues concerning the EEG NFT in sport performance include the how many sessions should be contained in training, what would be the ideal interval between each training, how to classify the responder and non-responder, and how long does the training effect last etc. Future studies are recommended to establish a systematical examination on the effects of SMR NFT to bridging the knowledge gap among issues above.

\section{References}

1. Vernon DJ (2005) Can neurofeedback training enhance performance? An evaluation of the evidence with implications for future research. Applied Psychophysiology Biofeedback 30(4): 347-364.

2. Mann CA, Sterman MB, Kaiser DA (1996) Suppression of EEG rhythmic frequencies during somato-motor and visuo-motor behavior. International Journal of Psychophysiology 23(1-2): 1-7.

3. Cheng MY, Hung CL, Huang CJ, Chang YK, Lo LC, et al. (2015) Expertnovice differences in SMR activity during dart throwing. Biol Psychol 110: 212-218.

4. Cheng MY, Wang KP, Hung CL, Tu YL, Huang CJ, et al. (2017) Higher power of sensorimotor rhythm is associated with better performance in skilled air-pistol shooters. Psychology of Sport and Exercise 32: 47-53.

5. Cheng MY, Huang CJ, Chang YK, Koester D, Schack T, et al. (2015) Sensorimotor rhythm neurofeedback enhances golf putting performance. Journal of Sport and Exercise Psychology 37(6): 626-636.

6. Hatfield BD, Hillman $\mathrm{CH}$ (2001) The psychophysiology of sport. Handbook of Sport Psychology, pp. 362-386.

7. Nan W, Rodrigues JP, Ma J, Qu X, Wan F, et al. (2012) Individual alpha neurofeedback training effect on short term memory. Int J Psychophysiol 86(1): 83-87. 